RUNNING HEAD: Neuroendocrine Patterns of Daily Stress

\title{
Psychological structure and neuroendocrine patterns of daily
}

\section{stress appraisals}

Maurizio Sicorello ${ }^{1}$, Andreas B. Neubauer ${ }^{2}$, Martin Stoffel $^{3}$, Friederike Koehler ${ }^{3}$, Andreas Voss $^{4}$, Beate Ditzen ${ }^{3}$

A published version can be found on https://doi.org/10.1016/j.psyneuen.2021.105198

${ }^{1}$ Heidelberg University, Medical Faculty Mannheim, Central Institute of Mental Health,

Department of Psychosomatic Medicine and Psychotherapy, K3 21, 68159 Mannheim,

Germany

${ }^{2}$ DIPF | Leibniz Institute for Research and Information in Education, Department for

Education and Human Development, Rostocker Str. 6, 60323 Frankfurt am Main, Germany

${ }^{3}$ Heidelberg University, University Hospital, Center for Psychosocial Medicine, Institute of Medical Psychology, Bergheimer Str. 20, 69115 Heidelberg, Germany

${ }^{4}$ Heidelberg University, Institute of Psychology, Department for Quantitative Research Methods, Hauptstraße 47-51, 69117 Heidelberg, Germany

Correspondence to:

Maurizio.sicorello@zi-mannheim.de

Clinic for Psychosomatic Medicine and Psychotherapy

Central Institute of Mental Health

J5, 68159 Mannheim, Germany

Declaration of interest: None 


\section{Highlights}

-Response surface analysis application to demand-resource mismatch in daily life

-Partial generalization of appraisal theories on a psychological level

-Robust associations between cortisol/alpha amylase and momentary daily life stress

-No consistent relation of cortisol/alpha amylase with threat-challenge appraisal 


\begin{abstract}
Threat and challenge are two fundamental appraisal concepts of psychological stress theories, determined by the mismatch between demands and resources. Previous research has predominantly investigated the neuroendocrine correlates of stress appraisal in laboratory contexts during acute demanding situations. We tested whether the psychoneuroendocrinology of stress appraisals can also be investigated in naturalistic trans-contextual everyday life settings. Forty-two participants produced five daily saliva samples and provided concurrent questionnaire data on subjective stress, demands, resources, and the threat-challenge continuum over the course of five days ( $69 \%$ female; mean age $=22.8$, range $=18-30$ years). Momentary salivary cortisol and alpha amylase were predicted with three-level autoregressive linear mixed models. We found that both momentary cortisol and alpha amylase were elevated during higher subjective stress. In contrast, cortisol was not significantly related to a bipolar threat-challenge indicator. Moreover within-person response surface analyses showed no effect of the mismatch between demands and resources on either physiological stress indicator, but confirmed theoretically proposed effects on subjective threat-challenge, which was replicated in another intensive longitudinal $(N=61)$ and a large cross-sectional sample $(N=1194)$. In sum, our study (a) suggests robust relations between subjective stress and HPA/SAM axis activity on a moment-to-moment basis and (b) confirms theoretical predictions concerning stress appraisal and the mismatch between demands and resources on a psychological level. In contrast, no neuroendocrine patterns of threat-challenge were found, suggesting that neuroendocrine patterns might be context-specific and do not apply to a general demandresource imbalance in everyday life.
\end{abstract}

Keywords: stress; appraisal; cortisol; alpha amylase; ambulatory assessment; response surface analysis 


\section{Psychological structure and neuroendocrine patterns of daily stress appraisals}

\section{Introduction}

\subsection{Theoretical background}

Stress - as an unspecific adaptive response to internal or external demands (Selye, 1974) — is essential to our understanding of human well-being and performance. However, despite its unspecific core, not all kinds of stressful experiences are identical. Psychological theories mostly distinguish between two anticipatory appraisals which shape our experience of stress: threat and challenge (Blascovich, 2008; Lazarus and Folkman, 1984). Identifying the psychological determinants and physiological patterns that distinguish threat and challenge is critical to understand how stress appraisal and its physiology relate to differential health outcomes.

Threat and challenge were originally conceptualized as anticipatory appraisals of situational demands against personal resources (Blascovich, 2008; Lazarus and Folkman, 1984). In previous theoretical formulations, both appraisals call for anticipatory coping efforts, but while threat focuses on anticipated losses and is often accompanied by negative emotions, challenge focuses on the potential for gain and growth.

A long tradition of laboratory studies has almost unequivocally considered the hypothalamic-pituitary-adrenomedullary (HPA) axis as a central part of the stress response, but its specific involvement in threat and challenge appraisals is less clear and different hypotheses have been proposed. The biopsychosocial model of threat and challenge (BPSM; Blascovich, 2008; Tomaka, Blascovich, Kelsey, \& Leitten, 1993) posited that threat and challenge result in distinct activation patterns of the sympatho-adreno-medullary (SAM) and the HPA axes: While the SAM axis is posited to be active during both threat and challenge, representing a general effort component, only threat appraisals are hypothesized to activate the HPA axis (Blascovich, 2008; Seery, 2011). The appraisal specificity of the HPA-axis for threat proposed by the BPSM 
is in opposition to an appraisal general view on HPA axis activity, which assumes an unspecific HPA response to any kind of demand on the organism (Selye, 1974). A third possibility is that both appraisals involve the HPA axis, but to a different degree, with higher responses during threat (appraisal asymmetry; e.g. Gaab, Rohleder, Nater, \& Ehlert, 2005). Notably, there is currently no conclusive evidence supporting either hypothesis (Denson et al., 2009; Ennis et al., 2001; Gaab et al., 2005; Poppelaars et al., 2019; Quested et al., 2011).

The research summarized above largely focused on concrete anticipatory appraisals preceding discrete motivated performance situations, most often involving active coping and a social evaluative component. However, arguably, appraisal systems might be constantly active during our daily lives: Individuals might appraise situational demands and personal resources in an ongoing fashion, accompanied by continuous co-fluctuations of the associated neuroendocrine systems to facilitate appropriate allostatic levels. In this vein, studies from daily life have demonstrated associations of salivary cortisol (sCORT) with the occurrence of minor daily life stressors, subjective stress, and negative affective states on a momentary basis (for a review, see Schlotz, 2019). It remains, however, an open question if there are discrete neuroendocrine patterns associated with momentary threat and challenge appraisals in daily life, similarly to what has been posited for motivated performance situations under controlled laboratory settings.

\subsection{Present study and hypotheses}

To test the relation of HPA and SAM axis activity with momentary stress appraisal, we conducted an ambulatory assessment study in which participants collected five daily saliva samples over five days and reported on their current demands, resources, subjective stress, and stress appraisal using a bipolar threat-challenge scale (Turner et al., 2014). This intensive longitudinal approach (Bolger and Laurenceau, 2013) allows to model within-person relations with sufficient statistical power for smaller effect sizes than comparable between-person designs (Arend and Schäfer, 2018) under naturalistic conditions in everyday life. 


\subsubsection{Psychological hypotheses}

Before the neuroendocrine analyses, we tested whether our self-report measures of momentary subjective stress and threat-challenge show relations with demands and resources conforming to what might be expected from theories on appraisals in acute stress situations as typically targeted in laboratory studies.

Hypothesis 1: Overall psychological stress is a consequence of the discrepancy between demands and resources according to the transactional stress theory, regardless of the absolute size of demands (Lazarus, 1999).

Hypothesis 2: Threat and challenge are determined by the balance between demands and resources, while absolute demands must be high for either appraisal to occur (Blascovich, 2008; Lazarus, 1966, p. 89; Lazarus, 1999, p. 77; Lazarus \& Folkman, 1984, p. 36) ${ }^{1}$. If resources outweigh demands, a challenge appraisal is more likely; if demands outweigh resources, a threat appraisal is more likely.

For these analyses, we used response surface analysis (RSA), which allows for a detailed assessment of hypotheses concerning the imbalance between demands and resources and which alleviates methodological shortcomings of previous approaches (i.e., difference scores and ratios; Edwards, 2002; see section 2.4.2). RSAs on self-report measures were repeated in another intensive longitudinal and a large cross-sectional sample to assess direct replicability and generalizability to between-person studies. A translation of the predictions in hypotheses 1 and 2 to RSA is provided in section 2.4.2 and depicted in Figure 1.

\subsubsection{Neuroendocrine hypotheses}

For the neuroendocrine analyses, we predicted momentary sCORT and alpha amylase (sAA) from subjective stress and a bipolar threat-challenge item. sAA served as an indicator of SAM activity (Ditzen, Ehlert, \& Nater, 2014; Nater \& Rohleder, 2009).

\footnotetext{
${ }^{1}$ Lazarus and Folkman $(1984$, p. 43) noted that primary and secondary appraisal do not have a sequential order, but rather are complex and mixed processes, which cannot be viewed separately and are not necessarily conscious.
} 
Hypothesis 3: We expected sCORT to be elevated during phases of higher subjective stress (Schlotz, 2019).

Hypothesis 4: For sCORT and the threat-challenge continuum, we competitively tested the three neuroendocrine appraisal hypotheses derived from laboratory studies: (4a) Appraisal specificity predicts an approximately linear relation between the threat-challenge scale and sCORT, with higher sCORT concentrations during threat. (4b) Appraisal generality predicts a quadratic (U-shaped) relation (elevation towards both extremes). (4c) Appraisal asymmetry predicts both a linear and a positive quadratic relation, resulting in an asymmetric U-shape with larger sCORT elevations for threat.

Hypothesis 5: We expected sAA to be elevated during phases of higher subjective stress (Nater et al., 2005), as for sCORT.

Hypothesis 6: In line with the BPSM predictions for motivated performance situations, we expected sAA to be elevated during both challenge and threat appraisal.

\section{Methods}

\subsection{Participants}

The sample was recruited over a participant database of the Psychological Institute at Heidelberg University, Germany. Of 60 university students who completed an initial online screening, nine did not meet the inclusion criteria and further nine did not attend their scheduled appointment, resulting in a final sample of $n=42(69 \%$ female; Mean age $=22.8, S D=3.3)$. Given the sample size and number of measurements, the design has sufficient statistical power $(\geq .80)$ to detect small within-person effect sizes in the area of $r \approx .17$ (Arend \& Schäfer, 2018). Applicants were included if, according to their self-reports, they (1) were between 18 and 40 years old, (2) were right-handed, (3) were nonsmokers, (4) had no chronic physical disease, (5) had no psychiatric or neurological disorders, (6) were not pregnant, (7) did not regularly take medication or hormonal contraceptives, (8) did not have surgery during the preceding three 
months, and (9) possessed a smartphone with the android operating system (version 4.0 or higher). Most participants were German native speakers (88.1\%). Non-native speakers had adequate German language skills to understand instructions and follow the study protocol. They were offered $100 €$ as an incentive with $15 €$ as an optional bonus if they filled out more than $90 \%$ of the ambulatory assessment questionnaires. All participants provided written informed consent prior to the study procedures. The study was approved by the ethics committee of the Faculty of Behavioural and Cultural Studies at Heidelberg University.

Information on the longitudinal $(N=61)$ and cross-sectional $(N=1194)$ replication samples for the psychological relations can be found in the supplements.

\subsection{Ambulatory Assessment}

2.2.1 Procedure. Participants came to a personal appointment where the movisensXS-app (version 1.0.0 and version 1.1.0, movisens GmbH, Karlsruhe, Germany) was installed on their personal phones to receive the ambulatory assessment questionnaires. The study procedure was explained to them orally and in written form. They informed us on their intended wake up time on the next five days to schedule the assessments according to their individual daily routine. Participants received 21 questionnaires each day, over the course of those next five days. The first daily measurement taken upon participant awakening was discarded for the present analyses, as it did not contain items on threat-challenge, demands, or resources (it was collected for a separate project on post-awakening sCORT increase; Kramer et al., 2019). The remaining 20 daily questionnaires assessed the relevant psychological variables. A subset of five questionnaires per day prompted participants to collect saliva samples. Four of these five saliva samples followed a fixed schedule relative to the participant's wake up time $(+0.5 \mathrm{~h},+2.5 \mathrm{~h},+8 \mathrm{~h}$ and $+12 \mathrm{~h}$ ). The final saliva sample was initiated by the participants directly before they went to bed. This design enabled the study to cover inter- and intra-individual fluctuations of the diurnal rhythms of sCORT and sAA. The remaining 15 daily questionnaires were presented semi-randomized in the 14 hours after the morning survey (at least 25 minutes apart). 
Compliance was high, with participants completing $86.8 \%$ of questionnaires on average $(\mathrm{SD}=7.9 \%$, range $=65.7-97.1 \%)$. Participants returned on average $86.0 \%$ of the 25 relevant saliva samples together with a valid questionnaire $(\mathrm{SD}=13.2 \%$, range $=48.0-100 \%)$.

In the replication sample, participants received ten semi-randomized prompts (at least 30 minutes apart) each day between 9:30 a.m. and 9:30 p.m. for five consecutive days. No saliva samples were collected in this sample. One participant did not receive any questionnaires due to technical difficulties; two more participants were discarded due to low compliance (less than ten assessments; see Neubauer, Voss, \& Ditzen, 2018). After removing the two cases, average compliance was $77.6 \%(S D=15.4 \%$, range $=24-98 \%)$.

2.2.2. Psychological measures. Responses to all items were measured on visual analog scales (VAS) ranging from 0 to 100, with the numeric value invisible to the participant. Based on the BPSM literature, two unipolar items were constructed from a previous BPSM study to assess momentary demands and resources (Tomaka et al., 1993). One item measured current demands ("I experience my current demands as...") and one item measured current resources ("I experience my current resources to cope with these demands as..."). Scale anchors were very low (0) and very high (100).

A bipolar item ("I experience the current demands on me as a...") was used to assess an individual's position on the threat-challenge continuum (Turner et al., 2014). Scale anchors were threat (0) and challenge (100), with a line marking the middle of the scale. Participants who experienced neither threat nor challenge were expected to select the midpoint of the scale (displayed label: "neither"). In addition, items were included to measure to which extent participants felt "stressed", "uneasy/tense", "good/content", and "tired/exhausted" right now. Scale anchors were not at all (0) and very (100). Descriptive statistics and correlations are presented in Table 1.

The demand, resource, and threat-challenge items were used with the same wording in the replication and the cross-sectional samples, although partly on different scales. Only the 
threat-challenge scale in the intensive longitudinal replication sample had a range of 100. The remaining item responses were measured on 7-point Likert scales to give them the same response format as other items on the same page, which were not related to the current study. We transformed the scales to percent of maximum possible scores (POMP; Cohen, Cohen, Aiken, \& West, 1999) which have a range of 100 and facilitate comparisons with the main sample.

2.2.3. Saliva samples. Participants collected saliva samples during the five respective prompts using SaliCaps ${ }^{\circledR}$ (IBL, Hamburg, Germany). Participants were instructed to accumulate saliva for $60 \mathrm{~s}$ before drooling it into the tube using a plastic straw. Participants were asked to store the saliva samples in their fridge, until they were returned to the laboratory after the ambulatory assessment was completed and stored at $-80{ }^{\circ} \mathrm{C}$ for no more than six months before analysis. The concurrent questionnaires included additional covariates on whether participants consumed meals, drinks, and caffeine (binary indicators) as well as how physically active they have been (VAS; 0-100) since the last saliva sample (Adam and Kumari, 2009; Strahler et al., 2017).

\subsection{Analysis of SCORT and SAA}

All samples underwent one freeze-thaw cycle before analyses to break down mucopolysaccharides and to reduce fluid viscosity (Worthman et al., 1990). Using the manufacturers protocols, sCORT levels $(\mu \mathrm{g} / \mathrm{dL})$ were measured using an enzyme-linked immunosorbent assay (ELISA; RE52611, IBL International, Hamburg, Germany) while sAA (U/L) was measured using kinetic colorimetric assay with reagents from Roche Diagnostics (Mannheim, Germany). All samples were assayed in duplicates and mean values were calculated to be used in statistical analyses. Furthermore, all samples beyond the detection limits of the assays were excluded from analyses ( $n=14$ for sCORT; $n=11$ for sAA). Intraassay coefficients of variation $(\mathrm{CV})$ were $3.29 \%$ for sAA and $6.58 \%$ for sCORT. Inter-assay CVs were $4.94 \%$ for sAA and $7.45 \%$ for sCORT. All analyses were conducted at the biochemical lab at the Institute of Medical Psychology, University Hospital Heidelberg. 


\subsection{Statistical Analysis}

2.4.1 Multilevel analysis. The data has a hierarchical structure with measurements (level 1) nested within days (level 2), nested within participants (level 3). Multilevel analyses were performed in $\mathrm{R}$ (version 3.5.1) using the package nlme to accommodate the dependencies in the data with a first order continuous time autoregressive (CAR1) residual covariance structure on level 1. Time-varying level-1 and level-2 predictors were centered on the person mean to represent pure within-person effects (Brincks et al., 2017). The time indicator itself reflected hours since the first saliva sample on a given day. Level-3 covariates were grand mean centered. Random intercepts were modelled for both participants and days. Random slopes were included for the effect of time, due to the considerable individual differences in diurnal slopes (Schlotz, 2019), and for focal predictors which were immediately relevant to our hypotheses to control the type I error rate (Barr et al., 2013).

\subsubsection{Response surface analysis (RSA).}

To test the hypothesis that stress is the consequence of a mismatch or discrepancy between (high) demands and (low) resources with RSA, both demands and resources are included as individual predictors in a regression model. The RSA approach alleviates the shortcomings of approaches using ratios and difference scores. These shortcomings include (1) lower reliability, (2) nonlinearity/nonnormality in case of ratios, (3) ambiguous interpretation (e.g., a difference score blends two sources of variance and only one might be predictive), and (4) untested constraints (e.g., a hypothesis proposing a discrepancy effect of two variables makes the assumption that individual regression weights are equal in size and opposite in sign). For details on these limitations, see Edwards(2002), Nestler et al. (2019), or Shanock et al. (2010).

If stress is the consequence of a discrepancy between demands and resources (Lazarus, 1999), the regression coefficients of demands and resources have a comparable size but are opposite in signs, with demands increasing and resources decreasing stress. Additionally, in 
RSA the quadratic terms and interactions of demands and resources are added, which allows to test and illustrate more complex relations. If the discrepancy hypothesis is sufficient to predict subjective stress, the regression coefficients of all these higher order terms should not differ significantly from zero. This is illustrated in the upper left panel of Figure 1. The black line marks the response surface along the line of incongruent predictor values, which represents the discrepancy effect and is expected to have a linear positive slope (i.e. if demands more strongly outweigh predictors, higher values of subjective stress are predicted and vice versa).

Threat-challenge was theorized to be the consequence of a discrepancy between demands and resources, given absolute demands are high (Blascovich, 2008; Lazarus, 1999; Lazarus and Folkman, 1984). Response surfaces resemble the theoretical pattern most closely when there is a positive main effect of resources, an interaction between demands and resources, and a small quadratic effect of demands, but no main effect of demands and no quadratic effect of resources. This is illustrated in the left lower panel of Figure 1: The response surface along the line of incongruent predictor values is nonlinear, starting on the left side with a threatchallenge value of zero (neither threat nor challenge) and a slope of zero, which becomes more negative as demands increase. Hence, the black line here represents a nonexistent discrepancy effect at minimal demands and a maximal discrepancy effect at maximal demands.

We followed recent guidelines to adapt RSA to the multilevel setting (Nestler et al., 2019). For fixed effects, demands and resources were centered on their shared within-person mean. Then, their quadratic terms and interactions were added. Person means were added for the same effects to remove between-person variance. All models converged with random slopes for all predictors, except for the model which predicted threat-challenge in the intensive longitudinal replication sample, where random slopes could only be included for the main effects of demands and resources.

Response surface models in the cross-sectional sample followed the procedure described by Shanock and colleagues (2010). 
Theoretical model
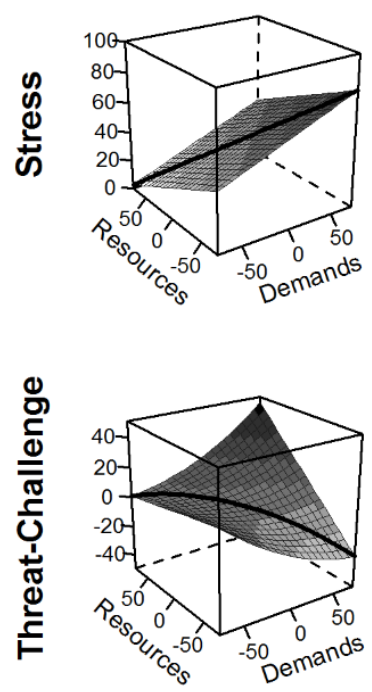

Main sample
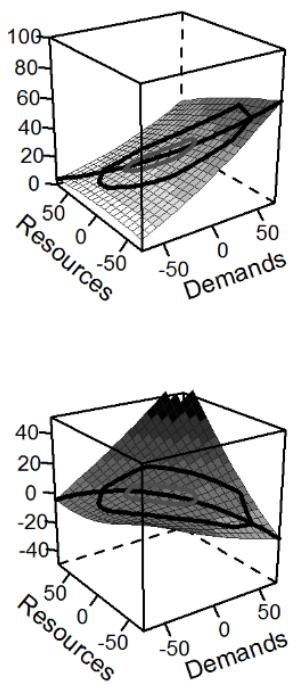

Replication
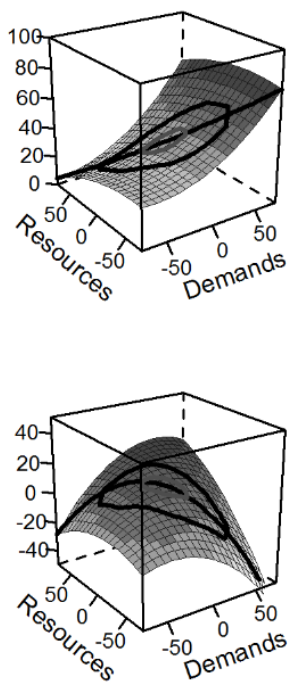

Cross-sectional
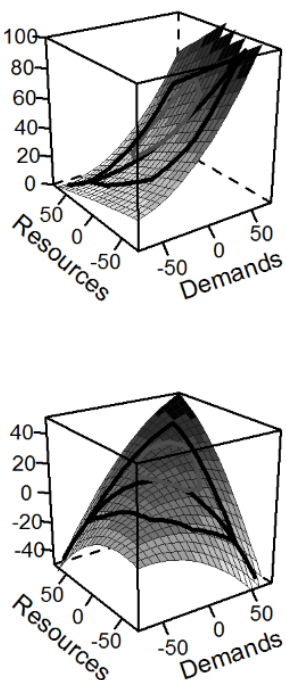

Figure 1. Response surfaces for subjective stress (upper row) and threat-challenge (lower row) predicted by demands and resources. The black line represents slope and curvature of the response surface along the line of incongruence, indicating the effect of the discrepancy between demands and resources on the outcome. In the plots based on empirical data, lightgrey circles on the response surfaces indicate the empirical range of $50 \%$ of the predictor values. The black circles on the response surfaces indicate the empirical range of $100 \%$ of the predictor values.

2.4.3. Endocrine response modelling. We followed the guidelines by Schlotz (2019) to model the endocrine response. In a first step, both sCORT and sAA were transformed by taking the natural logarithm to reduce the positive skew of the distribution. In a second step, the time series was detrended by including linear and quadratic fixed effects of time together with their random slopes to account for circadian rhythmicity and individual differences therein.

In a third step, additional covariates where added (Adam and Kumari, 2009; Schlotz, 2019; Strahler et al., 2017). Within-day covariates controlled for meals, drinks, caffeine, and 
physical activity since the last saliva sample. One day-level covariate captured the discrepancy between the scheduled first saliva sample and the actual self-reported time they woke up (e.g., when a participant woke up earlier). Another day-level covariate captured sleep quality of the last night's sleep, measured as the average of two items (“How well did you sleep?" and "How restful was your sleep?"). Age and gender were included as between-person covariates. We report Pseudo- $R^{2}$ for the within-person variance explained by the inclusion of covariates $(\mathrm{Xu}$, 2003).

In a last step, the explanatory variables were included. sCORT was predicted from the average of all self-reports since the last saliva sample to account for the delayed cortisol response, while sAA was predicted only from the concurrent self-report, given the short latency of the SAM response to stress (Nater et al., 2005). Neuroendocrine markers were predicted from subjective stress and threat-challenge in separate models. For subjective stress, only a linear effect was included. For threat-challenge, both a linear and a quadratic predictor were simultaneously added to test for appraisal generality.

$\mathrm{R}$ analysis scripts and supplements can be found on:

\section{https://osf.io/dkjsr/?view_only=15330187bc024882a039a476ef5a098d}

\section{Results}

\subsection{Self-Report Measures}

3.1.1 Correlations. To provide insights into the nomological net surrounding the stress and threat-challenge items used in this work, we examined bivariate correlations with indicators of contentment, tension, stress, and exhaustion.

On the within-person level, subjective stress was significantly correlated to all other measures (Table 1). It was most strongly associated with higher tension and worse mood. Interestingly, stress was only very weakly correlated to exhaustion/tiredness on a within-person level, but very strongly on a between-person level. Hence, people who on average experienced 
more subjective stress also on average felt more exhausted, but more subjectively stressful moments were practically unrelated to how exhausted one felt in that moment.

Threat-challenge was mostly related to other variables in a quadratic fashion (Figure 2). There appeared to be a hedonic optimum at slightly increased challenge levels in terms of momentary stress, mood, and tension. In contrast, exhaustion decreased linearly when participants moved from the threat to the challenge end of the item.

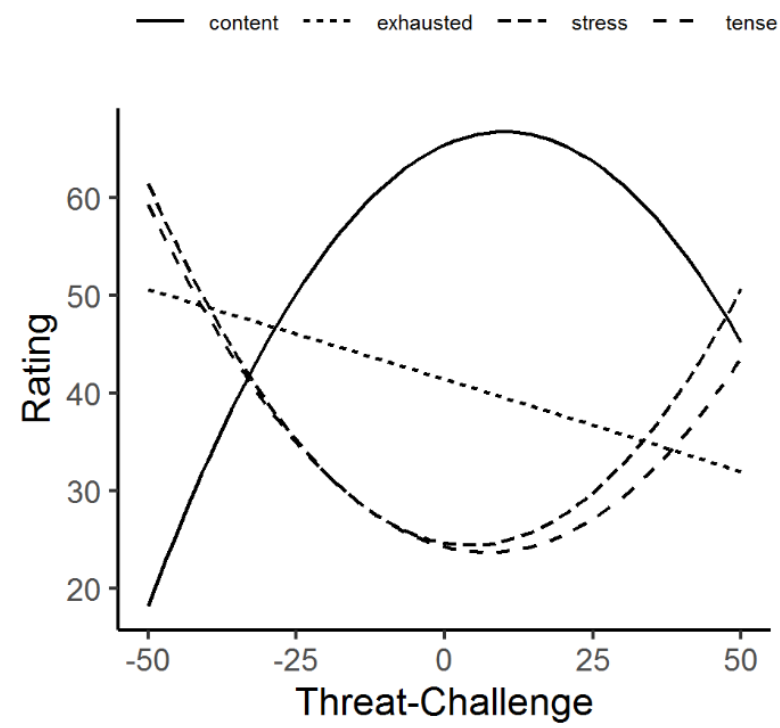

Figure 2. Within-person relations between threat-challenge and other self-reports.

3.1.2 RSA for subjective stress (Hypothesis 1). The transactional stress theory states that stress is a consequence of the discrepancy between demands and resources. This was reflected in the response surface pattern (Figure 1). As expected, demands had a positive effect and resources a negative effect on subjective stress in the main sample, while the higher order terms were not statistically significant (Table 2). Nevertheless, the effect of demands was substantially larger than the effect of resources and the confidence intervals of their absolute effect sizes did not overlap. This is inconsistent with a pure discrepancy effect, which implies similar absolute effect sizes for both predictors. Our data suggest that the concurrent experience of high levels of both demands and resources will still be associated with stress, even if there is 
no discrepancy between them. The same pattern was found in the replication sample and the cross-sectional sample, with the exception that in these other two samples the quadratic effect of demands became statistically significant as well, accelerating the effect of demands for higher values. This nonlinear effect was small in the replication sample, but relatively large in the cross-sectional sample. In sum, subjective stress self-reports were dominated by current demands and moderately decreased by current resources in all samples.

5.1.3 RSA for threat-challenge (Hypothesis 2). Appraisal theories propose that demanding situations will be more likely experienced as challenge if resources outweigh demands and as threat if demands outweigh resources (Blascovich, 2008; Lazarus, 1999; Lazarus and Folkman, 1984). The necessity of high demands (in absolute terms) is the critical difference to the conceptualization of stress as a mere mismatch between demands and resources. The empirical response surface of the main sample conformed to these assertions (Figure 1). As predicted, the effects of resources and the demand $\times$ resource interaction were significantly related to the threat-challenge continuum (Table 2).

Contrary to our expectations, there was a significant main effect of demands, but this main effect was not found in the replication sample. Moreover, the quadratic effect of demands was not significantly different from zero in the main sample and even had a significantly negative effect in the opposite direction in the replication and cross-sectional samples. Additionally, there was an unexpected negative quadratic effect of resources in both the intensive longitudinal and the cross-sectional replication samples. This led to a pronounced symmetrical inverted U-shape of the line of incongruence depicted in Figure 1, with threat appraisals also becoming more likely when resources strongly outweighed demands. Nevertheless, the black boundaries on the response surface indicate that this counterintuitive configuration only occurs at extrapolated values not represented in the data of the intensive longitudinal replication sample.

\subsection{Neuroendocrine response}


3.2.1 sCORT. The sCORT levels had a mean of $-1.76\left(S D_{\text {between }}=0.24\right.$ and $\left.S D_{\text {within }}=0.98\right)$. The circadian rhythm accounted for $68.8 \%$ of the within-person variance and is depicted in Figure S1 in the supplement. Adding the remaining covariates accounted for another $7.2 \%$ of within-person variance. The full output of the covariate model is presented in Table S1.

3.2.1.1 sCORT and subjective stress (Hypothesis 3). Subjective stress since the previous saliva sample significantly predicted higher momentary sCORT $(\gamma=0.006, S E=0.002,95 \%$ $\mathrm{CI}=[0.002,0.009], p=.001$; Figure 3A). Standardizing the regression coefficient on the within-person $S D$ s, this corresponds to $\gamma_{\mathrm{s}}=.10$.

As sCORT samples at $+0.5 \mathrm{~h}$ are often used to calculate the cortisol awakening response, this particular saliva sample might differ to some degree from the remaining samples. Rerunning the model without the $+0.5 \mathrm{~h}$ saliva samples led to similar results $(\gamma=0.007$, $S E=0.002,95 \% \mathrm{CI}=[0.002,0.011], p=.005)$. Moreover, there is evidence that earlier awakening times are associated with higher cortisol output throughout the day (Edwards, Evans, Hucklebridge, \& Clow, 2001). In our study, self-reported awakening time varied with a standard deviation of 1.33h around a mean of 8:06 a.m. Supporting the findings by Edwards and colleagues (2001), earlier self-reported awakening times were associated with significantly higher sCORT concentrations on the same day in the covariate model $(\gamma=-0.06, S E=0.021$, $95 \% \mathrm{CI}=[-0.10,-0.02], p=.003)$. Still, the effect of subjective stress remained stable in terms of approximate effect sizes and statistical significance in both the main model $(\gamma=0.005$, $S E=0.002,95 \% \mathrm{CI}=[0.002,0.010], p=.004)$ and the model without the $+0.5 \mathrm{~h}$ saliva samples $(\gamma=0.006, S E=0.002,95 \% \mathrm{CI}=[0.001,0.011], p=.011)$.

3.2.1.2 sCORT and threat-challenge (Hypothesis 4). Neither the linear nor the quadratic effect of threat-challenge were significant for sCORT (linear: $\gamma=-0.002, S E=0.002,95 \%$ $\mathrm{CI}=[-0.005,0.002], p=.228 ;$ quadratic: $\gamma=0.000, S E=0.00012,95 \% \mathrm{CI}=[-0.0002$, 0.0003], $p=.743$; Figure 3B). Again, rerunning the model without the $+30 \mathrm{~min}$. saliva samples 
and/or self-reported awakening time as a covariate led to similar results, with all $\gamma \approx-0.002$ and $p>.22$ for the linear trend and all $\gamma \approx 0.000$ and $p>.87$ for the quadratic trend.

A recent simulation demonstrated that significance tests in random slope models can lead to conservative results when true slope variance is very small (Matuschek et al., 2017). The authors suggested to include random slopes if they improved the model fit. A likelihood ratio test between a model with and without random slopes for the two threat-challenge predictors was not significant $\left(\chi^{2}(9)=4.32, p=.889\right)$. BIC and AIC were larger in the random slope model, indicating a worse parsimony-corrected model fit. A model without random slopes resulted in a larger and statistically significant U-shaped quadratic effect of threat-challenge $(\gamma=0.0002, S E=0.00008,95 \% \mathrm{CI}=[0.00001,0.0003], p=.043)$. However, this model could be overly liberal and should be interpreted with caution. Therefore, figure 3B depicts the more conservative full random slope model.

Past physiological studies on threat and challenge appraisal have assumed that the mismatch between demands and resources - either in terms of a ratio or difference score - can be used to operationalize the threat-challenge continuum, given that laboratory tasks are usually demanding. These approaches have several limitations, which can be resolved with RSA (see 2.4.2). As we found that demands and resources influenced threat-challenge, but threatchallenge was not related to sCORT, we also explored the relation between sCORT and the mismatch between demands and resources directly using RSA. There were no significant within-person relations between sCORT and any of the demand/resource predictors (all $p>$ 0.077), leading to a flat response surface (Figure S2).

Taken together, sCORT appeared to be robustly associated with the amount of subjective stress since the last measurement but was not preferentially determined by threat or challenge according to either the threat-challenge item or the RSA analysis on the mismatch between demands and resources. 
3.2.2 $\mathrm{sAA}$. The $(\log ) \mathrm{sAA}$ response had a mean of $4.25\left(S D_{\text {between }}=0.81\right.$ and $\left.S D_{\text {within }}=0.71\right)$. The circadian rhythm accounted for $32.8 \%$ of the within-person variance and is depicted in Figure S3. Adding the remaining covariates accounted for another $8.0 \%$ of within-person variance. The full output of the covariate model can be found in Table S2.

3.2.2.1 sAA and subjective stress (Hypothesis 5). Momentary subjective stress significantly predicted higher concurrent sAA $(\gamma=0.003, S E=0.001,95 \% \mathrm{CI}=[0.001,0.006], p=.012)$. Standardizing the regression coefficient on the within-person $S D$ s, this corresponds to a $\gamma_{\mathrm{s}}$ of .08 (Figure 3C).

3.2.2.2 sAA and threat-challenge (Hypothesis 6). Neither the linear nor the quadratic effect of threat-challenge were significant for sAA (linear: $\gamma=-0.001, S E=0.002,95 \%$ $\mathrm{CI}=[-0.004,0.002], p=.449 ;$ quadratic: $\gamma=0.0001, S E=0.00007,95 \% \mathrm{CI}=[-0.0002$, 0.0000], $p=.091$ ). As for sCORT, there were no significant within-person relations between sAA and any of the demand/resource predictors (all $p>0.085$ ), again leading to a flat response surface (Figure S4). 

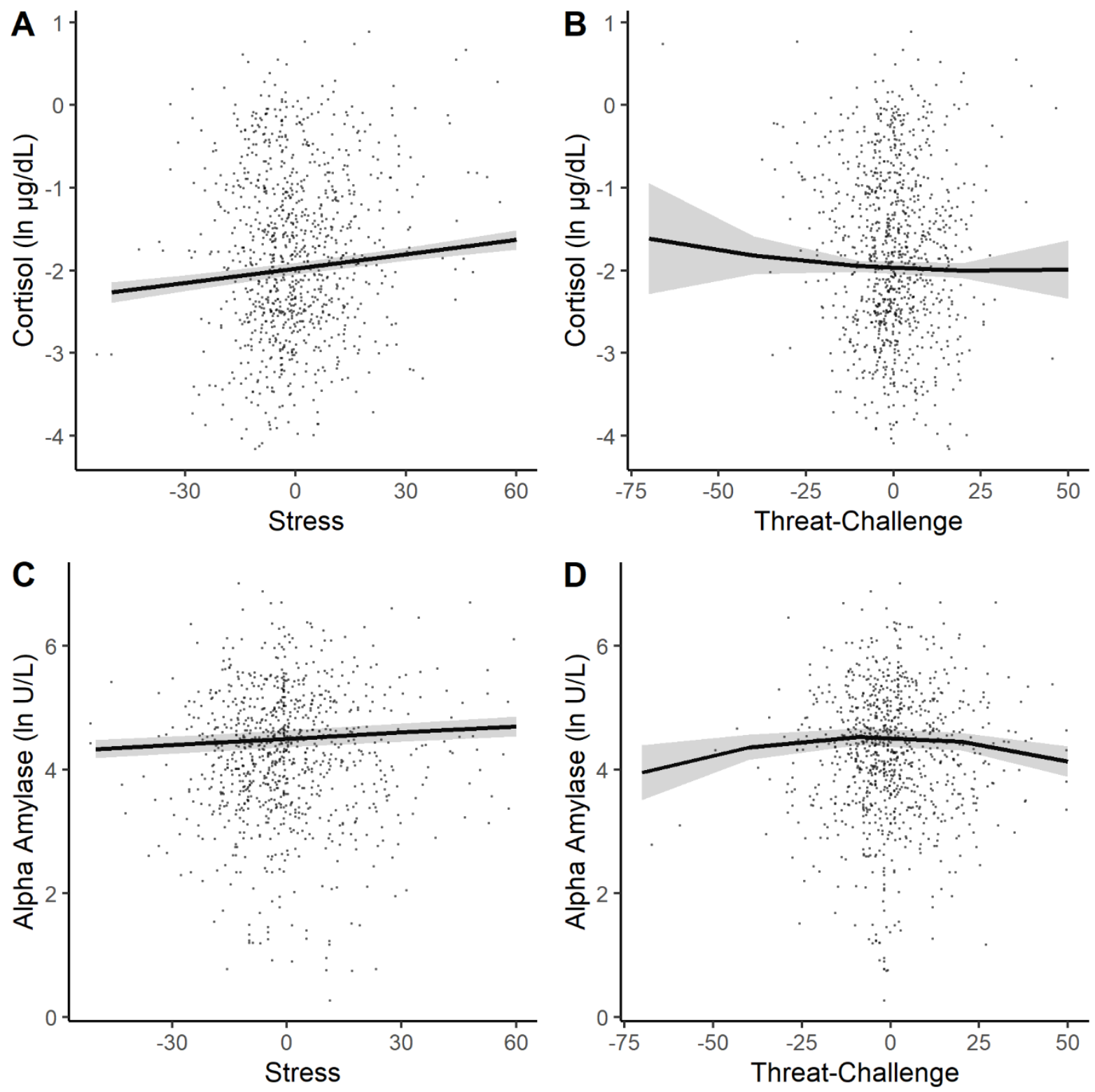

Figure 3. A: Momentary salivary cortisol predicted by subjective stress since the last saliva sample. B: Momentary salivary cortisol predicted by threat-challenge appraisal since the last saliva sample. Larger values represent a stronger challenge appraisal. C: Momentary salivary alpha amylase predicted by concurrent subjective stress. D: Momentary salivary alpha amylase predicted by concurrent threat-challenge appraisal. Larger values represent a stronger challenge appraisal. Neuroendocrine measures were log transformed. The grey areas around regression lines represent $95 \%$ confidence bands.

\section{Discussion}


Early on, researchers acknowledged stress as an essential part of life which is not simply always harmful and to be avoided, but also bears the potential to positively affect health, life satisfaction, and personal growth (Selye, 1974). Threat and challenge appraisals are likely candidates to explain these differential effects of stressful experiences. The current study presents analyses on the psychological structure and neuroendocrine responses associated with overall subjective stress and specific appraisals of threat and challenge on a moment-to-moment basis in everyday life.

\subsection{Psychological relations}

We used RSA to validate our measures of subjective stress and of the threat vs. challenge appraisal based on established psychological stress theories concerning the role of the mismatch between demands and resources. This application of RSA addresses shortcomings of past approaches which employed differences or ratios of demands and resources (see 2.4.2) and has the potential to reveal more complex relations. Our data overall matched predictions derived from psychological appraisal theories. These results were also largely confirmed in the replication and the cross-sectional sample, demonstrating generalizability to between-person designs. There were, however, also slight but reliable inconsistencies with the transactional stress theory.

Although subjective stress was largely determined by current demands and resources, it was not a result of the pure discrepancy between the two, only partially supporting hypothesis 1. When the difference between demands and resources is assumed to be the sole cause of stress, roughly equal effect sizes for both measures would be expected. However, in our data, in all three samples demands had a greater impact on stress than resources. A possible interpretation is that while resources do have a protective effect, people will still feel subjectively stressed when facing high demands if resources are not considerably higher, contrary to Lazarus' popular seesaw metaphor (Lazarus, 1999). It is unclear whether these discrepancies point towards a lack of generalizability to unspecific daily life stressors, imperfections in 
measurement, or might even hold in laboratory settings, which could profit from employing the RSA approach described here.

Our results regarding the threat-challenge continuum largely conformed to the theoretical positions of the transactional stress theory and the BPSM, largely confirming hypothesis 2: The effect of incongruence between demands and resources became stronger for higher demands. That is, in situations of high demands, if demands outweighed resources, people appraised the current situation as a threat, whereas if resources outweighed demands, they appraised the situation as a challenge. If demands were low, the situation was perceived as neutral (neither threat nor challenge).

Notably, in the replication sample the appraisal also shifted towards threat when very low demands were accompanied by very high resources. However, as these constellations of demands and resources were not present in the actual data, this finding is based on extrapolation and needs to be interpreted with caution. Moreover, we found that the threat-challenge continuum cannot be simply collapsed on a bipolar valence continuum, in line with previous arguments (Blascovich, 2008). Rather, low to moderate challenge appeared to be a hedonic optimum in terms of contentment, tension, and stress, while only momentary exhaustion distinguished clearly between threat and challenge, with high challenge being associated with the lowest feelings of exhaustion.

Additionally, we observed different within- and between-subject associations of subjective stress and tension/exhaustion. Participants who on average experienced more subjective stress also feel more exhausted. From an intrapersonal perspective, however, more stressful moments did not coincide with increased exhaustion felt at that moment. This is consistent with the notion that chronic stress leads to adverse effects on well-being, but momentary bouts of stress may activate energy resources. This highlights that trait-like measures can be insufficient to address certain questions in stress research and emphasizes the 
importance of intensive longitudinal ambulatory assessment studies to target within-person effects (Molenaar and Campbell, 2009).

\subsection{Neuroendocrine response}

For sCORT, we found a robust positive effect of self-reported stress (hypothesis 3) consistent with past daily life studies (Schlotz, 2019). For the threat-challenge continuum (hypothesis 4), results were less clear cut. Neither the linear nor the quadratic effects of threatchallenge were significantly related to sCORT in our main analyses (which included random slopes for both terms). Figure 3B suggests a tendency for a positive quadratic effect, which became significant only in a model without the random slope for threat-challenge. This second analysis was conducted post-hoc because models with random slopes that are not supported by the data might be too conservative (Matuschek et al., 2017), while models without these random slopes might be too liberal (Barr et al., 2013). In terms of the competing hypotheses (appraisal generality; appraisal specificity, appraisal asymmetry) these results appear to favor appraisal generality of the HPA axis (hypothesis 4a). Ultimately, however, there was no strong support for either of these hypotheses in context-independent naturalistic daily life experiences. This underlines the importance of the situational constraints imposed by previous theories, as it suggests that neuroendocrine patterns observed in motivated performance situations or similarly defined circumstances (e.g., laboratory settings) may not necessarily transfer to everyday stress experiences. Our findings suggest that patterns predicted by the BPSM might require active task engagement and an instrumental goal-directed response and might therefore not be observed in generic everyday situations as targeted in the present work.

For sAA, we also found a robust association with subjective stress in daily life (hypothesis 5). Still, we did not find sAA to be elevated towards the ends of the threat-challenge continuum (hypothesis 6). Past physiological studies on stress appraisal most commonly operationalized the threat-challenge continuum as the ratio or difference between perceived demands and resources (Ennis et al., 2001; Poppelaars et al., 2019). An RSA of sCORT and 
sAA showed no effects of demands, resources, or their discrepancy, resulting in flat response surfaces. These null findings are again consistent with the idea that stronger situational constraints might be necessary to observe physiological patterns accompanying threat and challenge.

Another possible explanation for these findings pertains to the specific neuroendocrine response component we targeted in the present study. The BPSM was inspired by Dienstbier's (1989) position that a strong and short sympathetic response is a marker of physiological toughness. Similarly, for the HPA axis, not only the peak but also the recovery rate is increasingly discussed as a marker of adaptive stress responses (Fiksdal et al., 2019), which could serve as an additional outcome when the cortisol response is measured at a higher frequency.

\subsection{Limitations}

While our ambulatory assessment design provides the opportunity to investigate the within-person relations between stress appraisal and its neuroendocrine correlates in a transcontextual naturalistic manner, it also comes with several limitations.

First, experiments afford a higher control over the measurement context and the temporal components of neuroendocrine responses. We tried to address these issues by including relevant covariates based on past guidelines (Adam and Kumari, 2009; Schlotz, 2019; Strahler et al., 2017) and compensate for the lag in the cortisol response by predicting it from subjective stress since the previous saliva sample. Nevertheless, effect sizes are likely diluted compared to laboratory studies, which might be partly alleviated by the statistical power gained from the within-subject design (Arend and Schäfer, 2018). Notably, the robust relations between subjective stress and cortisol/alpha amylase confirm the existence of detectable neuroendocrine signals in ambulatory assessments (Schlotz, 2019).

Second, research on transactional stress theory and BPSM have predominantly focused on concrete demanding situations, which often require active instrumental behavior and include 
a social evaluative component, whereas we did not assess characteristics of external events. Thus, in contrast to BPSM and transactional stress theory, our study treats stress appraisals as continuously varying evaluative states which occur throughout daily life and are associated with responses of allostatic systems. In the reported response surfaces, moments of high absolute demands would reflect typical laboratory task characteristics more closely, but they still rest on the subjective assessment of participants and are not necessarily coupled with a specific stressor. Thus, these high demands could also be caused by other factors, such as repeated hits or even by worries regarding the future, thereby possibly anticipating upcoming demands. Therefore, in our study, we generalize a core concept of stress theories to an everyday life context, where the cause of demands can include - but is not restricted to - distinct external stressors. This is an important distinction to make when interpreting the results of the present study. While assessing concrete situational characteristics in daily life would be an important extension of our study, these assessments would still rely on subjective evaluations. The objective control of situational characteristics remains a crucial advantage of laboratory studies, with the disadvantage that individuals cannot easily be assessed in a broad range of naturalistic settings. In our view, experimental and ambulatory assessment approaches are not competing, but instead provide a complementary perspective. Still, additional measurement of situational characteristics and discrete stressful events would be an important addition to the research design used here.

Third, we only tested a sample of healthy young students, which might limit generalizability. This might also restrict the range of stressful and threatening environments our participants experienced, although Table 1 and Figure 3 indicate substantial variance, especially for subjective stress, which had a within-person standard deviation close to the symmetrically distributed mood item "good/content". Threat-challenge, in turn, showed stronger evidence for insufficient variance, which might have hindered the detection of associations to neuroendocrine outcomes. This might be addressed by restricting analyses to high-stress or 
motivated performance situations in future studies, necessitating a longer assessment period to gather enough events.

Last, we used single-item measurements of the relevant concepts. This was done to allow for a higher sampling frequency, to use the original and most common items in research on the BPSM (Tomaka et al., 1993), and provide commensurability between measured demands and responses, which is a central prerequisite for response surface analysis (Edwards, 2002). Still, there is merit in employing longer scales which use a different conceptualization of threat and challenge (e.g. Gaab, 2009), although we are not aware of a scale that treats threat and challenge as opposite ends of a continuum. Viewing them on a continuum might be an oversimplification, but it also affords high testability of hypotheses concerning the physiological patterns which distinguish the two appraisals.

\subsection{Conclusion}

Our results add to the current understanding of stress by emphasizing several points: First, everyday momentary subjective stress was not a strict mismatch between perceived demands and resources, but instead dominated by perceived demands rather than resources. Second, although threat appraisals appear to be an undesirable state from a hedonic perspective, challenge is not its desirable antithesis. Instead, low to moderate challenge states appeared to be most hedonically desirable. Third, cortisol and alpha amylase were unspecifically elevated during higher subjective stress, with no apparent specificity for either threat or challenge appraisal. Hence, neither biomarker can be directly equated with negatively toned distress in everyday stress situations. Fourth, the mismatch between demands and resources is at the core of many stress-related psychological theories, but the corresponding hypotheses have been mostly relying on ratios and difference scores, which is methodologically problematic. We demonstrated that RSA might be a fruitful statistical tool to address these questions, which could also be adopted in laboratory settings. Last, while many relations relevant to stress might be similar among within- and between-person levels, we also found notable differences. 
Looking at the intrapersonal structure of hypothesized causal networks is therefore indispensable for future stress research, facilitating the triangulation with cross-sectional research. 


\section{Acknowledgments}

This work was supported by a grant from German Excellence Initiative DFG funds to Heidelberg University (ZUK 49/252.152) to BD and AV. The funding source was neither involved in the design of the study; in the collection, analysis and interpretation of data; in the writing of the report; nor in the decision to submit the article for publication. 


\section{References}

Adam, E.K., Kumari, M., 2009. Assessing salivary cortisol in large-scale, epidemiological research. Psychoneuroendocrinology 34, 1423-1436. https://doi.org/10.1016/j.psyneuen.2009.06.011

Arend, M.G., Schäfer, T., 2018. Statistical Power in Two-Level Models: A Tutorial Based on Monte Carlo Simulation. Psychol. Methods 24, 1-19. https://doi.org/10.1037/met0000195

Barr, D.J., Levy, R., Scheepers, C., Tily, H.J., 2013. Random effects structure for confirmatory hypothesis testing: Keep it maximal. J. Mem. Lang. 68, 255-278. https://doi.org/10.1016/j.jml.2012.11.001

Blascovich, J., 2008. Challenge and threat, in: Elliot, A.J. (Ed.), Handbook of Approach and Avoidance Motivation. Psychology Press, New York, NY, pp. 431-445.

Bolger, N., Laurenceau, J.-P., 2013. Intensive longitudinal methods: An introduction to diary and experience sampling research. Guilford Publications Inc., New York, NY.

Brincks, A.M., Enders, C.K., Llabre, M.M., Bulotsky-Shearer, R.J., Prado, G., Feaster, D.J., 2017. Centering Predictor Variables in Three-Level Contextual Models. Multivariate Behav. Res. 52, 149-163. https://doi.org/10.1080/00273171.2016.1256753

Buwalda, B., Scholte, J., de Boer, S.F., Coppens, C.M., Koolhaas, J.M., 2012. The acute glucocorticoid stress response does not differentiate between rewarding and aversive social stimuli in rats. Horm. Behav. 61, 218-226. https://doi.org/10.1016/j.yhbeh.2011.12.012

Cohen, P., Cohen, J., Aiken, L.S., West, S.G., 1999. The Problem of Units and the Circumstance for POMP. Multivariate Behav. Res. 34, 315-346. https://doi.org/10.1207/S15327906MBR3403_2

Denson, T.F., Spanovic, M., Miller, N., 2009. Cognitive Appraisals and Emotions Predict Cortisol and Immune Responses: A Meta-Analysis of Acute Laboratory Social Stressors 
and Emotion Inductions. Psychol. Bull. 135, 823-853. https://doi.org/10.1037/a0016909

Dienstbier, R.A., 1989. Arousal and physiological toughness: Implications for mental and physical health. Psychol. Rev. 96, 84-100. https://doi.org/10.1037/0033-295X.96.1.84

Ditzen, B., Ehlert, U., Nater, U.M., 2014. Associations between salivary alpha-amylase and catecholamines - A multilevel modeling approach. Biol. Psychol. 103, 15-18. https://doi.org/10.1016/j.biopsycho.2014.08.001

Edwards S, Evans P, Hucklebridge F, Clow A (2001): Association between time of awakening and diurnal cortisol secretory activity. Psychoneuroendocrinology 26: 613622. https://doi.org/10.1016/S0306-4530(01)00015-4Edwards, J.R., 2002. Alternatives to difference scores: Polynomial regression analysis and response surface methodology, in: Drasgow, F., Schmitt, N. (Eds.), The Jossey-Bass Business \& Management Series. Measuring and Analyzing Behavior in Organizations: Advances in Measurement and Data Analysis. Jossey-Bass, pp. 350-400. https://doi.org/10.1037/e576892011-020

Ennis, M., Kelly, K.S., Lambert, P.L., 2001. Sex differences in cortisol excretion during anticipation of a psychological stressor: Possible support for the tend-and-befriend hypothesis. Stress Heal. 17, 253-261. https://doi.org/10.1002/smi.904

Fiksdal, A., Hanlin, L., Kuras, Y., Gianferante, D., Chen, X., Thoma, M. V., Rohleder, N., 2019. Associations between symptoms of depression and anxiety and cortisol responses to and recovery from acute stress. Psychoneuroendocrinology 102, 44-52. https://doi.org/10.1016/j.psyneuen.2018.11.035

Gaab, J., 2009. PASA Primary Appraisal Secondary Appraisal. Verhaltenstherapie 19, 114115. https://doi.org/10.1159/000223610

Gaab, J., Rohleder, N., Nater, U.M., Ehlert, U., 2005. Psychological determinants of the cortisol stress response: the role of anticipatory cognitive appraisal.

Psychoneuroendocrinology 30, 599-610. https://doi.org/10.1016/j.psyneuen.2005.02.001

Hummel, E.M., Hessas, E., Müller, S., Beiter, T., Fisch, M., Eibl, A., Wolf, O.T., Giebel, B., 
Platen, P., Kumsta, R., Moser, D.A., 2018. Cell-free DNA release under psychosocial and physical stress conditions. Transl. Psychiatry 8. https://doi.org/10.1038/s41398-0180264-X

Jimeno, B., Hau, M., Verhulst, S., 2018. Corticosterone levels reflect variation in metabolic rate, independent of 'stress.' Sci. Rep. 8, 1-8. https://doi.org/10.1038/s41598-018$31258-\mathrm{Z}$

Kramer, A.C., Neubauer, A.B., Stoffel, M., Voss, A., Ditzen, B., 2019. Tomorrow's gonna suck: Today's stress anticipation predicts tomorrow's post-awakening cortisol increase. Psychoneuroendocrinology 106, 38-46. https://doi.org/10.1016/j.psyneuen.2019.03.024

Lazarus, R.S., 1999. Stress and emotion: A new synthesis. Springer, New York, NY.

Lazarus, R.S., 1966. Psychological stress and the coping process. McGraw-Hill, New York, NY.

Lazarus, R.S., Folkman, S., 1984. Stress, appraisal, and coping. Springer, New York, NY.

Matuschek, H., Kliegl, R., Vasishth, S., Baayen, H., Bates, D., 2017. Balancing Type I error and power in linear mixed models. J. Mem. Lang. 94, 305-315. https://doi.org/10.1016/j.jml.2017.01.001

Molenaar, P.C.M., Campbell, C.G., 2009. The New Person-Specific Paradigm in Psychology. Curr. Dir. Psychol. Sci. 18, 112-117. https://doi.org/10.1111/j.1467-8721.2009.01619.x Nater, U.M., Rohleder, N., 2009. Salivary alpha-amylase as a non-invasive biomarker for the sympathetic nervous system: Current state of research. Psychoneuroendocrinology 34, 486-496. https://doi.org/10.1016/j.psyneuen.2009.01.014

Nater, U.M., Rohleder, N., Gaab, J., Berger, S., Jud, A., Kirschbaum, C., Ehlert, U., 2005. Human salivary alpha-amylase reactivity in a psychosocial stress paradigm. Int. J. Psychophysiol. 55, 333-342. https://doi.org/10.1016/j.ijpsycho.2004.09.009

Nestler, S., Humberg, S., Schönbrodt, F.D., 2019. Response Surface Analysis With Multilevel Data : Illustration for the Case of Congruence Hypotheses 24, 291-308. 
Neubauer, A.B., Voss, A., Ditzen, B., 2018. Exploring need dynamics within and across days in everyday life: A three-level analysis. J. Res. Pers. 77, 101-112. https://doi.org/10.1016/j.jrp.2018.10.001

Poppelaars, E.S., Klackl, J., Pletzer, B., Wilhelm, F.H., Jonas, E., 2019. Social-evaluative threat: Stress response stages and influences of biological sex and neuroticism. Psychoneuroendocrinology 109, 104378. https://doi.org/10.1016/j.psyneuen.2019.104378

Quested, E., Bosch, J.A., Burns, V.E., Cumming, J., Ntoumanis, N., Duda, J.L., 2011. Basic psychological need satisfaction, stress-related appraisals, and dancers' cortisol and anxiety responses. J. Sport Exerc. Psychol. 33, 828-846. https://doi.org/10.1123/jsep.33.6.828

Schlotz, W., 2019. Investigating associations between momentary stress and cortisol in daily life: What have we learned so far? Psychoneuroendocrinology 105, 105-116. https://doi.org/10.1016/j.psyneuen.2018.11.038

Seery, M.D., 2013. The Biopsychosocial Model of Challenge and Threat: Using the Heart to Measure the Mind. Soc. Personal. Psychol. Compass 7, 637-653. https://doi.org/10.1111/spc3.12052

Seery, M.D., 2011. Challenge or threat? Cardiovascular indexes of resilience and vulnerability to potential stress in humans. Neurosci. Biobehav. Rev. 35, 1603-1610. https://doi.org/10.1016/j.neubiorev.2011.03.003

Selye, H., 1974. Stress: Lebensregeln vom Entdecker des Stress-Syndroms [Stress without distress]. Rowohlt, Hamburg.

Shanock, L.R., Baran, B.E., Gentry, W.A., Pattison, S.C., Heggestad, E.D., 2010. Polynomial Regression with Response Surface Analysis: A Powerful Approach for Examining Moderation and Overcoming Limitations of Difference Scores. J. Bus. Psychol. 25, 543554. https://doi.org/10.1007/s10869-010-9183-4 
Strahler, J., Skoluda, N., Kappert, M.B., Nater, U.M., 2017. Simultaneous measurement of salivary cortisol and alpha-amylase: Application and recommendations. Neurosci. Biobehav. Rev. 83, 657-677. https://doi.org/10.1016/j.neubiorev.2017.08.015

Tomaka, J., Blascovich, J., Kelsey, R.M., Leitten, C.L., 1993. Subjective, physiological, and behavioral effects of threat and challenge appraisal. J. Pers. Soc. Psychol. 65, 248-260. https://doi.org/10.1037/0022-3514.65.2.248

Turner, M.J., Jones, M. V., Sheffield, D., Barker, J.B., Coffee, P., 2014. Manipulating cardiovascular indices of challenge and threat using resource appraisals. Int. J. Psychophysiol. 94, 9-18. https://doi.org/10.1016/j.ijpsycho.2014.07.004

Worthman, C.M., Stallings, J.F., Hofman, L.F., 1990. Sensitive salivary estradiol assay for monitoring ovarian function. Clin. Chem. 36, 1769-1773.

https://doi.org/10.1093/clinchem/36.10.1769

Xu, R., 2003. Measuring explained variation in linear mixed effects models. Stat. Med. 22, 3527-3541. https://doi.org/10.1002/sim.1572 
Table 1

Descriptive statistics and correlations for momentary self-report measures

\begin{tabular}{|c|c|c|c|c|c|c|c|c|c|c|c|}
\hline Measure & $M$ & $S D_{\text {between }}$ & $S D_{\text {within }}$ & 1 & 2 & 3 & 4 & 5 & 6 & 7 & 8 \\
\hline 1. Stress & 26.67 & 15.38 & 16.53 & - & .11 & $.62 * * *$ & $.59 * * *$ & $-.40 * *$ & $.90 * * *$ & $.68 * * *$ & $-.42 * *$ \\
\hline $\begin{array}{l}\text { 3. Squared Threat- } \\
\text { Challenge }\end{array}$ & 317.0 & 286.8 & 392.21 & $.27 * * *$ & $.33^{* * *}$ & - & $.69 * * *$ & $-.31 *$ & $.60^{* * *}$ & $.49 * *$ & $-.36^{*}$ \\
\hline 5. Resources & 61.08 & 17.11 & 14.08 & $-.15^{* * *}$ & $.22 * * *$ & $-.15^{* * *}$ & $-.12 * * *$ & - & & & \\
\hline 6. Uneasy/Tense & 26.27 & 15.85 & 16.66 & $.63 * * *$ & $-.17 * * *$ & $.24 * * *$ & $.43 * * *$ & $-.17 * * *$ & - & $.64 * * *$ & $-.40 * *$ \\
\hline 7. Tired/Exhausted & 41.60 & 12.44 & 21.6 & $.06 * * *$ & $-.16^{* * *}$ & -.01 & .02 & $-.15^{* * *}$ & .07 & - & $-.50 * *$ \\
\hline
\end{tabular}

Note. The correlation matrix shows Pearson correlation coefficients within participants below the diagonal and between participants above the diagonal, calculated with the statsBy function of the psych package (v1.8.12).

$* p<.05, * * p<.01, * * * p<.001$ 
Table 2

Polynomial regressions of subjective stress and threat-challenge appraisal on the mismatch between demands and resources across three samples

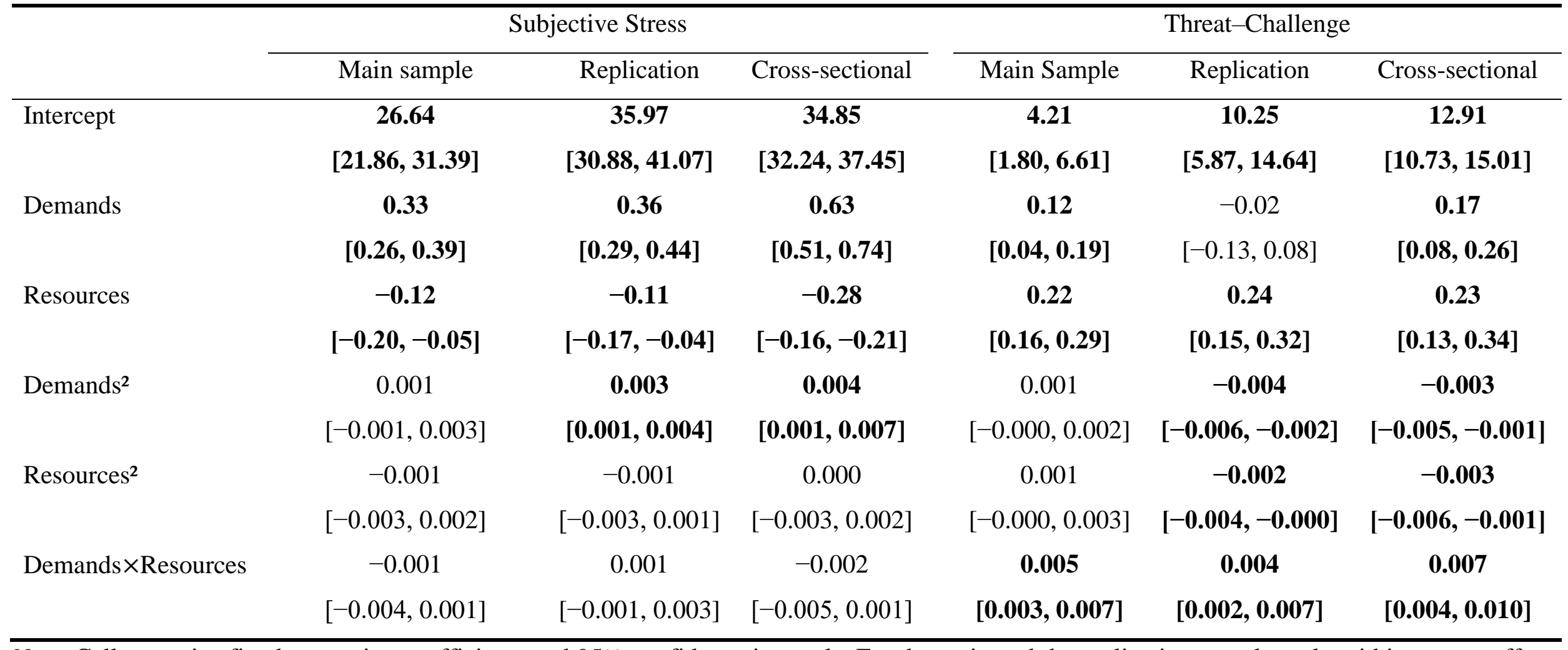

Note. Cells contains fixed regression coefficients and 95\% confidence intervals. For the main and the replication sample, only within-person effects are shown.

Significant effects $(p<.05)$ are shown in bold 
Neuroendocrine Patterns of Daily Stress 